\title{
Spring JDBC Data Access on Hemo-biological Indices of Monitoring
} System

\author{
Xiuying Guo ${ }^{1, a}$, Lixia Wang ${ }^{2, b}$ \\ ${ }^{1}$ Department of Sports Training, Hebei Institute of Physical Education, Shijiazhuang, P.R. China \\ ${ }^{2}$ Physical Education College, Shijiazhuang University, Shijiazhuang, P.R. China \\ aguoxiuying555@souhu.com, bsmilexia1225@163.com
}

Keywords: hemo-biological indices; monitoring system; Spring JDBC; data access

\begin{abstract}
Blood storage the information on human health, many diseases need to test blood index. Physiological and the pathological changes of the organism often cause the changes on blood component, blood biological indexes monitoring has important significance to improve health level and prevent diseases. This paper is based on the access to Spring JDBC research data to support the development of monitoring system in hemo-biological indices. First, describe the monitoring index; Then, has carried on the data logical structure design based on SQL Server database management system ;Finally, according to the steps about the Spring JDBC data access to complete the entity class structure, the preparation of DAO implementation and configuration of the transaction manager. The study on the data access in this paper has the characteristics of simple and general, which can improve the development efficiency of system and maintenance.
\end{abstract}

\section{Introduction}

The blood is an organization which circulating flow in the circulation system, heart and blood vessel cavity. Blood is a kind of connective tissue, composed of plasma and blood cells. Blood contains a variety of nutrients, have function on nutrition organizations, regulate organ activities and defense the deleterious substance. Blood storage the information on human health, many diseases need to test blood index. Physiological changes and the pathological changes of the organism is often caused by a blood component changes, so the detection of blood composition have important clinical significance [1]. In sports training, the application of hemo-biological indices pay more and more attention to the athletes about body function evaluation and the training load monitoring, but at present the research on the use of biological indicators about comprehensive monitoring on the functional state of athletes is less than before [2]. The evaluation through the comprehensive indicators can accurately grasp the players to adapt to the situation of sports training load, reflect the functional state of athletes, which provides an objective basis for improving the effect of training and sports performance.

Data access is the basic work of the development process of management system. JDBC (Java Data Base Connectivity) is a kind of Java API used to execute the SQL statement, can provided data access method for various of relational database. Spring is an open source framework, the core technology is a lightweight container, for the development of enterprise application based on J2EE to provide full range of support for the application framework [3]. When based on Spring framework to develop the Java application, there are two kinds of data access methods, one is Spring JDBC, another is Spring Hibernate. This paper is based on the access to Spring JDBC research data to support the development of monitoring system in hemo-biological indices.

\section{Monitoring Indices Description}

In recent years the hemo-biological indices commonly used include hemoglobin ( $\mathrm{Hb})$, blood urea nitrogen (BUN), serum creatine kinase (CK), serum testosterone (T), serum cortisol $(C)$ and the ratio (T/C). The descriptions for each index are as follows:

(1) Hemoglobin (Hb). Hb is a protein that higher organisms is responsible for carrying oxygen, 
easy to combine with oxygen in high oxygen content places; and easy to separate from oxygen in areas where the oxygen content is low. Many reasons for high $\mathrm{Hb}$, it can be classified as elevated physiological and pathologist increase.

(2) The blood urea nitrogen (BUN). BUN determination is a auxiliary examination method used to check whether the urination function is normal. High BUN is the most common with renal dysfunction, including acute renal insufficiency and chronic renal insufficiency. And mainly is the damage of renal function which urea nitrogen can not be effectively discharged from the urine.

(3) Serum creatine kinase (CK). The response is reversibly catalyzed creatine and phosphocreatine generate adenosine triphosphate and two adenosine monophosphate. Positive reaction to oxidative phosphorylation generated ATP in the mitochondrial, enter the cell liquid in the form of creatine phosphate, to meet the needs of the cell physiological activities.

(4) Serum testosterone (T). $\mathrm{T}$ is the main male hormone. It is an important male hormone to maintain sex characteristics and sexual function. $\mathrm{T}$ is a certain fluctuation in daily, which usually higher in early morning than in the afternoon. This difference is bigger in adolescent, for comparison, the general requirements of blood time is required standard.

(5) Serum cortisol (C). C produced and secreted by the adrenal cortex, controlled by the adrenocorticotropic hormone on anterior pituitary. Secretion of adrenal cortex was reacted directly by measurement of $\mathrm{C}$. Higher than normal is adrenal hyperplasia, tumors, obesity, and intake of amphetamine.

(6) The ratio (T/C). Usually put the two together for functional evaluation, to reflect the metabolic process by the way of the change of T/C ratio. With the normal physiological conditions, the body can through own adjustment, make $\mathrm{T}$ and $\mathrm{C}$ balance, the ratio of the two is maintained in a certain range.

\section{Database Logical Structure Design}

The main task of the logical structure of the database design is to convert the conceptual data model for the organization of layer data model, namely according to the conceptual structure to derive for specific logical structure which database management system can handle [4]. In a relational model, two-dimensional table represents an entity set and its properties, using two dimensional table describes the connection between entity sets. Hemo-biological indices monitoring system uses SQL Server database management system, the logical structure in design shown as Table 1.

Table 1. Database logical structure on hemo-biological indices of monitoring system

\begin{tabular}{c|c|c|c|c}
\hline No & Name & Fields Type & Fields Width & Fields description \\
\hline 0 & ID & Decimal & 10,0 & Automatic increase \\
\hline 1 & PerNo & Varchar & 10 & Testing personnel number \\
\hline 2 & PerName & Varchar & 50 & Testing personnel name \\
\hline 3 & PerSex & Varchar & 2 & Testing personnel sex \\
\hline 4 & PerDB & Datetime & 4 & Personnel date of birth \\
\hline 5 & SamNo & Varchar & 20 & Data acquisition number \\
\hline 6 & SamDate & Datetime & 4 & Data acquisition date \\
\hline 7 & SamTime & Datetime & 4 & Data acquisition time \\
\hline 8 & SamEnv & Varchar & 500 & Data acquisition environment \\
\hline 11 & IndexHb & Decimal & 8,4 & Hemoglobin \\
\hline 12 & IndexBUN & Decimal & 8,4 & Blood urea nitrogen \\
\hline 13 & IndexCK & Decimal & 8,4 & Serum creatine kinase \\
\hline 14 & IndexT & Decimal & 8,4 & Serum testosterone \\
\hline 15 & IndexC & Decimal & 8,4 & Serum cortisol
\end{tabular}




\section{Spring JDBC Data Access}

JDBC Abstract framework provided by Spring included the core package (org.springframework.jdbc.core.JdbcTemplate), data package (org.springframework.jdbc.database.DriverManagerDataSource), object package (org.springframework.jdbc.object), support package (org.springframework.jdbc.support) etc.. Complete data access mainly consists of three steps.

Step 1: Entity class structure [5]. The entity class is commonly known as the POJO, mainly existed as the data management and business logic processing level classes, this class does not implement the interface under the special framework, only as a data container used for persistent storage of data. The entity class code is as follows:

\begin{tabular}{|c|c|}
\hline 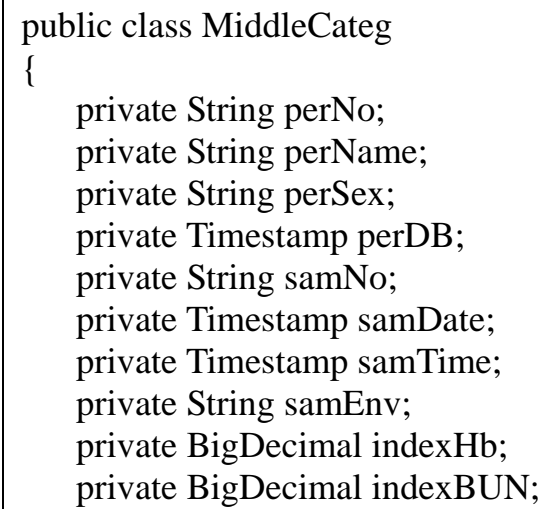 & $\begin{array}{l}\text { private BigDecimal indexCK; } \\
\text { private BigDecimal indexT; } \\
\text { private BigDecimal indexC; } \\
\text { private BigDecimal ratioTC; } \\
\text { public String getPerNo()\{ } \\
\text { return this.perNo; }\} \\
\text { public void setPerNo(String perNo)\{ } \\
\text { this.perNo = perNo; }\} \\
\text { //Omit the getter/setter method of the other } \\
\text { attributes } \\
\}\end{array}$ \\
\hline
\end{tabular}

Step 2: The preparation of DAO implementation. Data access object (DAO) is a design pattern of J2EE, to separate the business logic in high grade from data access logic in low level . DAO of Spring JDBC inherited from the JdbcDaoSupport class provided by Spring. Spring brings benefits to the DAO compilation, refinement of the data access exception and enriches the exception type; use the template callback mode, developers no longer write mode code and simplify programming. Based on DAO implementation of JDBC, to simplify the operation of the JDBC, access to JDBC objects are freed and transforming the exception type; the developer is responsible for the SQL, set the variable in the SQL and extract ResultSet. Limited to the length of paper, DAO implementation includes only a getHemobiologicalIndices class method, by calling this method can get all the information of hemo-biological indices. The realize code about DAO are as follows:

\begin{tabular}{|l|l|}
\hline $\begin{array}{l}\text { package cn.wode.springcore.jdbc; } \\
\text { import java.sql.ResultSet }\end{array}$ & $\begin{array}{l}\text { protected class HemobiologicalIndicesRowMapper } \\
\text { implements RowMapper \{ } \\
\text { import java.sql.SQLException; }\end{array}$ \\
$\begin{array}{c}\text { public Object mapRow(ResultSet rs, int rowNum) } \\
\text { import java.util.List; }\end{array}$ & $\begin{array}{l}\text { throws SQLException \{ } \\
\text { import javax.sql.DataSource; }\end{array}$ \\
import org.springframework.jdbc.core.RowMapper; & item.setPerNo(rs.getString("perNo")); \\
import & item.setPerName(rs.getString("perName")); \\
org.springframework.jdbc.core.support.JdbcDao & item.setPerSex(rs.getString("perSex")); \\
Support; & item.setPerDB(rs.getTimestamp("perDB")); \\
public class HemobiologicalIndicesDAO extends & item.setSamNo(rs.getString("samNo")); \\
JdbcDaoSupport implements & item.setSamDate(rs.getTimestamp("samDate")); \\
IHemobiologicalIndicesDAO & item.setSamTime(rs.getTimestamp("samTime")); \\
item.setSamEnv(rs.getString("samEnv")); & item.setIndexHb(rs.getBigDecimal("indexHb")); \\
public List getHemobiologicalIndices () \{ & item.setIndexBUN(rs.getBigDecimal("indexBUN")); \\
String sql="SELECT "perNo, perName, perSex, & item.setIndexCK(rs.getBigDecimal("indexCK")); \\
perDB, samNo, samDate, samTime, & item.setIndexT(rs.getBigDecimal("indexT")); \\
samEnv, indexHb, indexBUN, indexCK, & item.setIndexC(rs.getBigDecimal("indexC")); \\
indexT, indexC, ratioTC FROM & item.setRatioTC(rs.getBigDecimal("ratioTC")); \\
hemobiologicalIndices"; & return item;\} \\
return super.getJdbcTemplate().query (sql, new & \\
\hline
\end{tabular}


HemobiologicalIndicesRowMapper()); \}

Step 3: Configuration transaction manager. Based on the Spring IoC container, the Spring framework provides a function called lightweight transaction management. Different with common database transaction in an operation by the use of transaction management, Spring framework provides transaction management function is the method for the class in Java. The core framework of Spring transaction management consists of three interface definition, respectively is PlatformTransactionManager, TransactionDefinition and TransactionStatus. Among them, the PlatformTransactionManager interface defines two methods for transaction, the commit method is used to commit the transaction, the rollback method is used to roll back the transaction; the TransactionDefinition interface defines transaction strategy, can get the information on isolation level, dissemination, timeout, a category of business of transaction; TransactionStatus interface represents the state information of a transaction. Interface defines the methods to obtain the status of the transaction. The configuration of the transaction manager includes the data source configuration, configuration of transaction management object, configuration of transaction strategy, configuration $\mathrm{AOP}$ and so on.

\section{Conclusion}

With the wide application of modern information technology, the research object in the field of information of sports information and research field continues to expand, to prevent excessive fatigue of athletes or over training, and scientific regulation of athletes' competitive state before big games, and then create the best sports results in the competition, which are the new topics of information technology application in the field of sports science [6-7]. Many methods for monitoring the athletes' functions, in comparison, some biochemical components in body fluids can ideally reflect the determination of athlete's physiological state with a certain objectivity. Need to pay attention to monitoring functional state by blood biochemical indexes: to comprehensive evaluation should choose many indexes, relative to the unity of test conditions, pay attention to the individual difference of athletes and the characteristics of the project [8]. Spring JDBC data access has the characteristics of simple and general, and plays an important role in improving the development efficiency of monitoring system of hemo-biological indices and maintainability etc..

\section{References}

[1] Baidu Encyclopedia, "Blood," http://baike.baidu.com/view/18631.htm?fr=aladdin, 2014-11-20.

[2] M. Y. Lu, Z. J. Li, "Application of Hemobiological Indices to Monitor Functional State in Women Softball Players During their Winter Training," Journal of Changchun University of Science and Technology (Natural Science Edition), vol. 32, no. 3, pp. 424-426, 2009.

[3] J. Du, L. J. He, H. J. Ouyang "Design and Implementation of Online Examination System Based on JDBC and DAO Mode," Journal of Xianyang Normal University, vol. 29, no. 2, pp. 26-29, 2014.

[4] Baidu Encyclopedia, "Logical structure design of database," http://baike.baidu.com/view/3858534.htm?fr=aladdin, 2014-11-20.

[5] L. Zhang, W. X. Zhang, "Application of Vegetables Transportation and Sales Integrated Management System in Date Persistent Based on Spring and JDBC," Journal of Anhui Agricultural Sciences, vol. 40, no. 7, pp. 4401-4403, 2012.

[6] Y. Tian, Q. Wang, L. S. Feng, et al., "Monitoring of Sport Training and Regulation of Competitive State in Elite Athletes," China Sport Science, vol. 28, no. 9, pp. 3-11, 2008.

[7] W. Feng, W. T. Lin, X. L. Zhang, et al., "Competitive aerobics training course of physiological and biochemical monitoring and analysis," Journal of Mudanjiang Normal University(Natural Sciences Edition), vol. 38, no. 3, pp. 18-21, 2012.

[8] Y. Tan, "The integrated use of blood biological indexes monitoring functional state of athletes," Liaoning sports science and technology, vol. 33, no. 3, pp. 55-58, 2011. 